\title{
BMJ Open Which came first, obstructive sleep apnoea or hypertension? A retrospective study of electronic records over 10 years, with separation by sex
}

\author{
Eunjoo An, ${ }^{1}$ Michael R Irwin, ${ }^{2}$ Lynn V Doering, ${ }^{1}$ Mary-Lynn Brecht, ${ }^{1}$ \\ Karol E Watson, ${ }^{3}$ Ravi S Aysola, ${ }^{4}$ Andrea P Aguila, ${ }^{1}$ Ronald M Harper, ${ }^{5}$ \\ Paul M Macey (D) ${ }^{1}$
}

To cite: An E, Irwin MR, Doering LV, et al. Which came first, obstructive sleep apnoea or hypertension? A retrospective study of electronic records over 10 years, with separation by sex. BMJ Open 2021;11:e041179. doi:10.1136/ bmjopen-2020-041179

- Prepublication history for this paper is available online. To view these files, please visit the journal online (http://dx.doi org/10.1136/bmjopen-2020041179).

Received 02 June 2020 Revised 18 February 2021 Accepted 27 February 2021
Check for updates

(C) Author(s) (or their employer(s)) 2021. Re-use permitted under CC BY-NC. No commercial re-use. See rights and permissions. Published by BMJ.

For numbered affiliations see end of article.

Correspondence to

Dr Paul M Macey;

pmacey@ucla.edu

\section{ABSTRACT}

Objectives Obstructive sleep apnoea (OSA) is a risk factor for hypertension (HTN), but the clinical progression of OSA to HTN is unclear. There are also sex differences in prevalence, screening and symptoms of OSA. Our objective was to estimate the time from OSA to HTN diagnoses in females and males. Design Retrospective analysis of electronic health records (EHR) over 10 years (2006-2015 inclusive).

Setting University of California Los Angeles (UCLA) Health System in Los Angeles, California, USA.

Participants 4848 patients: females $n=2086$, mean (SD) age $=52.8$ (13.2) years; males $n=2762$, age $=53.8$ (13.5) years. These patients were selected from 1.6 million with diagnoses in the EHR who met these criteria: diagnoses of OSA and HTN; in long-term care defined by ambulatory visits at least 1 year prior and 1 year subsequent to the first OSA diagnosis; no diagnosis of OSA or HTN at intake; and a sleep study performed at UCLA.

Primary and secondary outcome measures The primary outcome measure in each patient was time from the first diagnosis of OSA to the first diagnosis of HTN (OSA to HTN days). Since HTN and OSA are progressive disorders, a secondary measure was the relationship between OSA to HTN time and age (OSA to $\left.\mathrm{HTN}=\beta_{1} \times \mathrm{Age}+\beta_{0}\right)$.

Results The median (lower and upper quartiles) days from OSA to HTN were: all $-532(-1439,-3)$; females -610 $(-1579,-42)$; and males $-451(-1358,0)$. Older age in both sexes was associated with less time to a subsequent HTN diagnosis or more time from a prior HTN diagnosis ( $\beta_{1}$ days/ year: all -16.9 , females -18.3 , males -15.9 ).

Conclusions HTN was on average diagnosed years prior to OSA, with a longer separation in females. Our findings are consistent with underscreening of OSA, more so in females than males. Undiagnosed OSA may delay treatment for the sleep disorder and perhaps affect the development and progression of HTN.

\section{INTRODUCTION}

Obstructive sleep apnoea (OSA), a breathing disorder that affects around 30 million people nationally, is a primary risk factor for hypertension (HTN). ${ }^{1}$ When comorbid with OSA, HTN is often treatment resistant. ${ }^{2}$ An accepted model
Strengths and limitations of this study

- Approximately 5000 is a large enough sample to provide reliable effect size and $\mathrm{Cl}$ calculations.

- Inclusion criteria ensure observations are from patients likely to be in regular contact with the University of California Los Angeles (UCLA) Health System, and hence to be in a position to be regularly screened for hypertension (HTN) and obstructive sleep apnoea (OSA).

- Separation by sex highlights the presence of clinical differences in patients with HTN and OSA, although the nature of the differences (eg, underscreening, or different progression in females and males) cannot be inferred.

- Limited generalisability due to data in a single health system and to the specific 10-year time period, the latter encompassing a change in diagnostic criteria for OSA.

- No data on diagnoses in other clinical settings or prior to 2006, meaning (1) many patients with HTN and OSA were likely excluded because the diagnoses were not recorded at UCLA, and (2) of the included patients some may have had prior diagnoses, thus affecting interpretation of the time from OSA to HTN measure.

is that OSA pathophysiology leads to HTN, ${ }^{3}$ implying that treating OSA would help prevent or resolve HTN. ${ }^{4}$ However, randomised trials of treating OSA with continuous positive airway pressure (CPAP) show modest effects on $\mathrm{HTN},{ }^{4}$ confirming links between the two conditions are not simplistic. Factors such as the association between rapid eye movement OSA and typical time time-of-night CPAP usage may explain the CPAP findings, ${ }^{5}$ but further data are needed to understand OSA-HTN relationships. Other relevant information is whether on average a diagnosis of OSA precedes a diagnosis of HTN. Despite the accepted pathophysiological model, there is little clinical evidence on the question 
of whether OSA or HTN appears first in the clinical record. Even though there are reasons other than disease progression that could influence the timing of diagnoses (differences in HTN and OSA screening, multifactorial nature of HTN), establishing the health record occurrence of HTN relative to OSA would contribute to our understanding of clinical and maybe mechanistic relationships between the conditions.

Assuming OSA is a precursor to HTN, we initially hypothesised that in patients with both conditions a diagnosis of OSA precedes a diagnosis of HTN. This hypothesis also assumes equivalent screening for both HTN and OSA, which, while unlikely to be true, nevertheless provides a starting point. Both HTN and OSA are progressive so clinical diagnoses usually occur months to years after condition onsets. Since screening for OSA is presumably less frequent than for HTN, the delay between onset and diagnosis likely differs between the two conditions, with OSA most likely having a longer delay than HTN. ${ }^{4}$ Hence, a finding that OSA precedes HTN clinically would strongly support the model of OSA preceding HTN; alternatively, if HTN precedes OSA diagnoses, we could not rule out a longer delay to OSA screening. In either case, quantifying the time between diagnoses would be useful.

Our objective was to test the hypothesis that OSA precedes HTN using electronic health records (EHR) within the University of California Los Angeles (UCLA) Health System. We assessed the time between OSA and HTN diagnoses in patients with both conditions. We also aimed to describe timing of diagnoses with respect to age and gender, since both factors influence prevalence and severity of both conditions. ${ }^{67}$

\section{METHODS}

We evaluated EHRs from the UCLA Health System over a 10-year period beginning January 2006. The patient records containing diagnoses of HTN or OSA were retrieved based on International Classification of Diseases diagnostic codes. Sleep study parameters and CPAP usage were not available. During the timeframe, the American Academy of Sleep Medicine scoring rules for respiratory events were updated ${ }^{89}$; the UCLA Sleep Disorders Center transitioned from the 1999 to 2012 rules in the year following their release. In patients with both conditions, the time from first diagnosis of OSA to first diagnosis of HTN was calculated. The month and day of the first encounter date was converted to 1 January to remove personal identifiers (date of visit), but otherwise times between diagnoses were accurate to within a day. The day of first OSA diagnosis was set as 0 (relative starting point), and the time difference represented as days to first HTN diagnosis. Sleep study parameters were not available in the EHR.

We calculated descriptive statistics of time from OSA to HTN, including the median which we tested for being nonzero with a sign test. The relationship between age and time from OSA to HTN was calculated using linear regression; although the data distribution was not expected to be normal, we chose the parametric model given the large sample size. We repeated these analyses for females and males separately. The location of diagnoses (outpatient vs inpatient) within the 10-year period affects possible OSA to HTN times, so we viewed these times separately by year and by gender. Statistics were calculated with MATLAB.

\section{Patient and public involvement}

The motivation to look at OSA and HTN was driven by authors' interactions with patients, including difficulties reported in getting the sleep disorder and comorbid conditions fully addressed. The sex-specific questions were motivated by female patients frequently reporting difficulty in being screened for OSA. Patients were not involved in the design or implementation or interpretation of the study.

\section{RESULTS}

Of the 1.6 million patient records evaluated ( $\mathrm{n}=1654067$ ) with at least one diagnosis, approximately 2\% (29 764) contained OSA diagnoses and 14\% (192 771) contained HTN diagnoses. There were $16974(1 \%)$ patient records with both diagnoses of OSA and HTN, of which 36\% (6124) had a sleep study within the UCLA Health System. (A sleep study is required to diagnose OSA.) We defined long-term care as patients with encounters at least a year before and after OSA diagnosis, who comprise 29\% (4848) of records of the patients with OSA/HTN. Data are available online. ${ }^{10}$

Table 1 shows the characteristics of OSA and HTN diagnoses and patient demographics, separated by gender. HTN was typically diagnosed earlier than OSA (median time difference -532 days). Females showed earlier HTN diagnosis relative to their OSA diagnosis than males (median days delay: females -610; males -451). Age was negatively related to time from OSA to HTN diagnosis in both sexes ( $\beta_{1}$ days/year: all -16.9 , females -18.3 , males -15.9 ); in other words, patients diagnosed with OSA at an older age had less time to a subsequent HTN diagnosis or more time since a prior HTN diagnosis. Findings are illustrated in figures 1 and 2.

We repeated these analyses for (1) the 16974 records of all patients with HTN and OSA diagnoses and (2) the 6124 with an UCLA sleep study but not meeting our definition of long-term care, and the direction of effects did not change for either set.

\section{DISCUSSION}

From 2006 to 2016 in the UCLA Health System, HTN was on average diagnosed years prior to OSA, with a longer separation in females. The time between diagnoses could reflect differences in screening, specifically that HTN is more frequently screened than OSA. Since there are proposed mechanisms by which OSA leads to HTN ${ }^{11}$ it is possible the condition of OSA precedes the condition of HTN, in which case these findings would reflect a delay in OSA relative to HTN screening of several years. Assuming better overall screening of HTN than OSA, the findings are also consistent with both conditions co-occurring, or with HTN preceding the development of OSA due to other factors. The present data cannot distinguish between these possibilities as both conditions share numerous risk factors, and it is therefore 
Table 1 Patient characteristics separated by gender

Time from OSA to HTN

\begin{tabular}{|c|c|c|c|c|}
\hline A & $\left(t_{\mathrm{O} \rightarrow \mathrm{H}}\right)$ days & All $(n=4848)$ & Females $(n=2086)$ & Males $(\mathrm{n}=2762)$ \\
\hline & $\begin{array}{l}\text { Mean (SD) } \\
(95 \% \mathrm{Cl})\end{array}$ & $\begin{array}{l}-732(1094.9) \\
(-764.6 \text { to }-701.8)\end{array}$ & $\begin{array}{l}-815.9(1127.3) \\
(-867.3 \text { to }-764.2)\end{array}$ & $\begin{array}{l}-668.6(1065.6) \\
(-708.1 \text { to }-626.8)\end{array}$ \\
\hline & Model by age (all $p<0.05) t_{\mathrm{O} \rightarrow \mathrm{H}}=\beta_{1} \times A g e+\beta_{0}$ & $\begin{array}{l}-16.9 \times \text { Age }+299.3 \\
\text { Model } F=221 \\
\beta_{1}: p<0.0001, t=-14.9\end{array}$ & $\begin{array}{l}-18.3 \times \text { Age }+301.2 \\
\text { Model } F=98 \\
\beta_{1}: p<0.0001, t=-9.9\end{array}$ & $\begin{array}{l}-15.9 \times \text { Age }+302.5 \\
\text { Model } F=125 \\
\beta_{1}: p<0.0001, t=-11.2\end{array}$ \\
\hline & \multicolumn{4}{|l|}{ Distribution } \\
\hline & HTN before OSA & $71.7 \%$ & $75.2 \%$ & $69.1 \%$ \\
\hline & $\begin{array}{l}\text { HTN with OSA } \\
\text { (within } 40 \text { days) }\end{array}$ & $7.0 \%$ & $5.9 \%$ & $7.9 \%$ \\
\hline & n before OSA & $81(92)$ & $98.4(99)$ & $67(83)$ \\
\hline & $\mathrm{n}$ after OSA & $218(211)$ & $248(223)$ & $195(199)$ \\
\hline B & Demographics & All $(n=4848)$ & Females $(n=2086)$ & Males (n=2762) \\
\hline & Age at OSA diagnosis, mean (SD) years & $53.3(13.4)$ & $52.8(13.2)$ & $53.8(13.5)$ \\
\hline & $\begin{array}{l}\mathrm{BMI}\left(\mathrm{m}^{2} / \mathrm{kg}\right) \\
\mathrm{n} \text { measurements }\end{array}$ & $\begin{array}{l}30.9(7.5) \\
n=3149 / 4848\end{array}$ & $\begin{array}{l}32.6(8.4) \\
n=1411 / 2086\end{array}$ & $\begin{array}{l}29.6(6.3) \\
n=1738 / 2762\end{array}$ \\
\hline & \multicolumn{4}{|l|}{ Race } \\
\hline & Native American /Pacific Islander & $<1 \%$ & $<1 \%$ & $<1 \%$ \\
\hline & Asian & $9.8 \%$ & $9.5 \%$ & $10.1 \%$ \\
\hline & Not Hispanic/Latino & $84.9 \%$ & $82.6 \%$ & $86.7 \%$ \\
\hline & Not specified & $2.2 \%$ & $1.2 \%$ & $3.0 \%$ \\
\hline
\end{tabular}

(A) Days from first OSA to first HTN diagnosis ( $\mathrm{t}_{\mathrm{O} \rightarrow \mathrm{H}}$ ); mean and $95 \% \mathrm{Cls}$, median, upper and lower quartiles (IQR) with sign test results, and regression model by age ( $F=$ overall model $F$ statistic; $t=\beta_{1} t$ statistic). (B) Demographics. Approximate age at time of OSA diagnosis. BMI was the closest recorded value to the date of OSA diagnosis; $n$ indicates subset of patients with BMI values. Race and ethnicity were as recorded in electronic health records.

BMI, body mass index; HTN, hypertension; OSA, obstructive sleep apnoea.

likely that there were multiple conditions that affected the diagnosis dates.

Considering possible common factors, the co-occurrence of OSA and HTN may be associated with structural and biological effects of obesity, which is highly prevalent in both conditions. ${ }^{3} 12$ In OSA, the extra layer of adipose tissue surrounding the neck may contribute to the narrowing of the upper airway, and rigidity of the thoracic and abdomen walls due to the adipose tissue deposits surrounding tissues, further compromising the air flow. ${ }^{13}$ In HTN, an increase in adipose tissue, especially surrounding the abdomen wall, may contribute to progression of HTN due to excess amount of adipokines produced. ${ }^{14} 15$ Adipokines play a key role in body homeostasis, including insulin regulation, lipid and glucose metabolism, coagulation, and angiogenesis and vascular remodelling, which all affect blood pressure regulation. ${ }^{15}$

The longer separation seen in females may be partially explained by more frequent screening in males due to differences in clinical presentation between the genders. For instance, women typically report fatigue, insomnia and depression, symptoms not classically associated with OSA, whereas men typically report daytime sleepiness, the defining symptom of the sleep disorder. ${ }^{16}$ Sex differences in OSA, such as structural changes in the $\operatorname{brain}^{17}$ and 


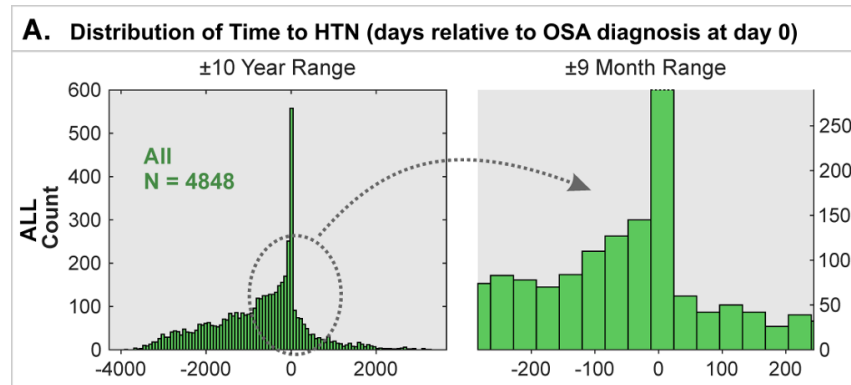

B. Time OSA-to-HTN by Age
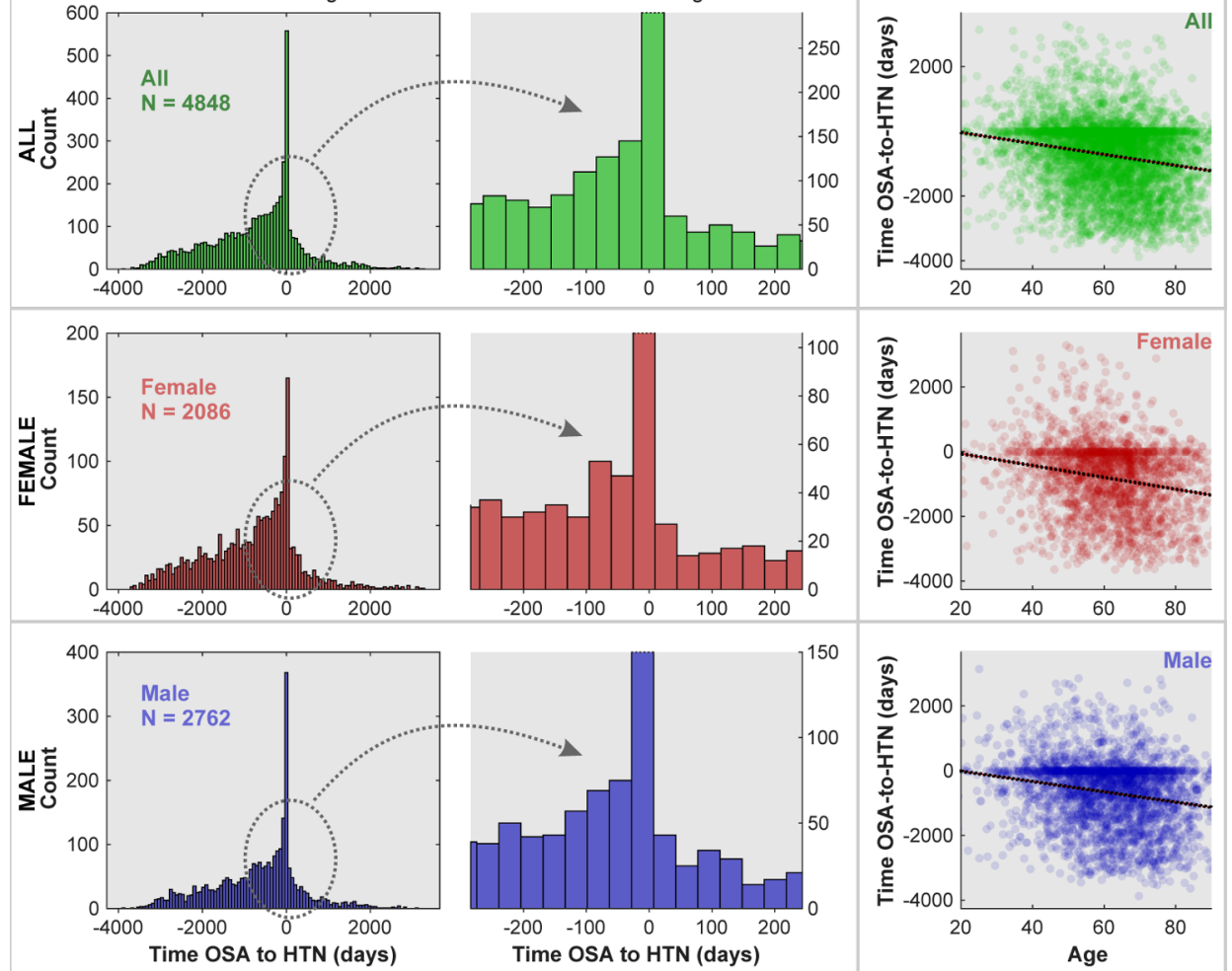

Figure 1 Time from obstructive sleep apnoea (OSA) to hypertension (HTN): distribution and time by age, separated by sex. (A) Distribution of time from OSA to HTN diagnoses, for combined ('ALL') and female and male patients; \pm 10 year and \pm 9 month ranges are shown. (B) Scatterplots of time from OSA to HTN diagnoses with respect to age at OSA diagnosis, for combined ('ALL') and female and male patients.

menopause ${ }^{18}$ mean that the OSA-HTN relationship may differ between males and females. Premenopausal women and postmenopausal women on hormone replacement therapy have shown significantly lower OSA prevalence, suggesting hormones may have a protective role in women. ${ }^{19}$ Other major differences between the sexes are that men have a higher prevalence of OSA than women by a factor of $2: 1{ }^{12}$ which could influence the likelihood of screening based on expectations that males are more likely to have the sleep condition.

No data were available on CPAP prescription or usage, and due to methodological complexities we did not consider

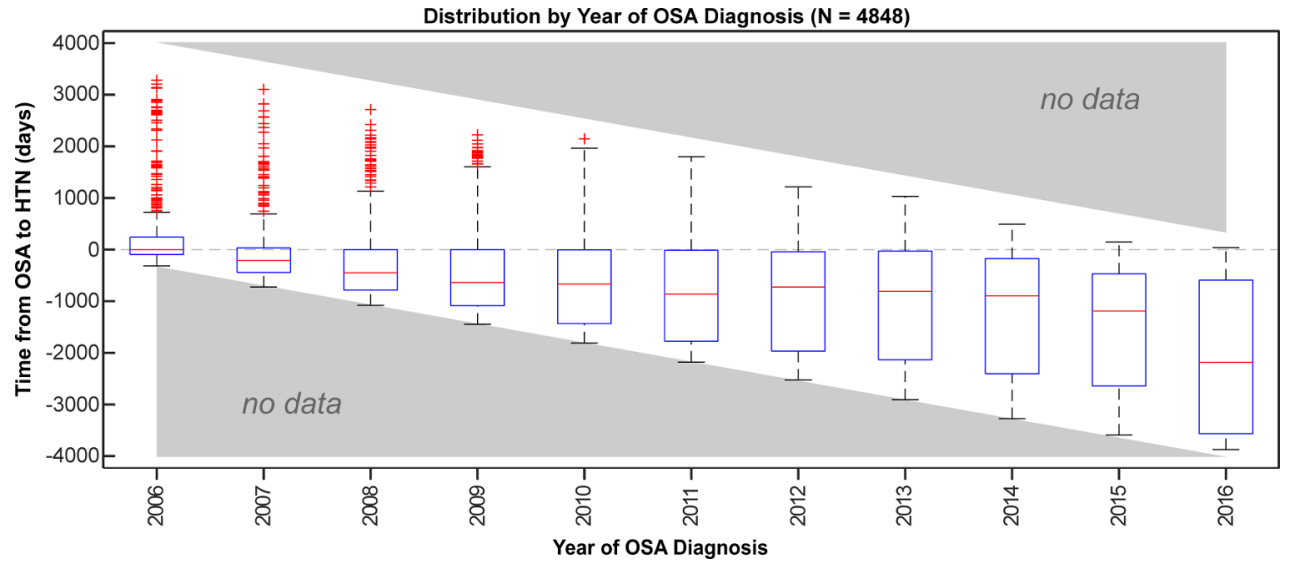

Figure 2 Distribution by year of obstructive sleep apnoea (OSA) diagnosis in patients with OSA and hypertension (HTN). Distribution by year of time from OSA to HTN diagnoses, with box plots by year of OSA diagnosis (box is between 25th and 75th percentiles, red line is median, whisker lines are ranges with outliers defined as more than 1 IQR from box marked as red '+'). Grey areas indicate no data are available for diagnoses prior to 2006 or after 2015 (so a delay from before 2006 or after 2015 could not be observed). Years are approximate ( \pm 6 months) as the referenced dates (first encounter) extracted from the electronic health records are set on 1 January, and were rounded to the middle of the year; thus the data for 2005 and 2016 reflect smaller numbers of patients. 
other comorbid conditions, so several possible interpretations remain for the present findings.

While these findings are from one health system and may not generalise, our analysis could be replicated with other EHR data sets. The analysis could also be replicated in future time periods, since screening for OSA may improve with time. OSA is a relatively recently discovered disease, and awareness has been increasing over the past two decades. ${ }^{12}$ Similarly, screening for HTN, perhaps with home testing, may lead to earlier diagnosis relative to onset, so the patterns seen from 2006 to 2016 may well differ in the future. Additionally, the present data and the refractory nature of HTN in CPAP-treated OSA suggest that the relationship of OSA to high blood pressure is complex. Any mechanisms by which OSA leads to HTN may be influenced by additional factors such as obesity and other comorbid conditions, or even bidirectional influences. ${ }^{20}$

\section{Author affiliations}

${ }^{1}$ UCLA School of Nursing, University of California, Los Angeles, Los Angeles, California, USA

${ }^{2}$ Department of Psychiatry and Biobehavioral Sciences, University of California, Los Angeles, Los Angeles, California, USA

${ }^{3}$ Department of Medicine, David Geffen School of Medicine at UCLA, University of California, Los Angeles, Los Angeles, California, USA

${ }^{4}$ Division of Pulmonary, Critical Care and Sleep Medicine, Department of Medicine, David Geffen School of Medicine at UCLA, University of California, Los Angeles, Los Angeles, California, USA

${ }^{5}$ Department of Neurobiology, University of California, Los Angeles, Los Angeles, California, USA

Contributors EA and PMM conceived and designed the study, and performed the analyses. MLB assisted with the statistical analysis. MRI, LVD, KEW, RSA and RMH interpreted the findings based on their expertise in cardiology, sleep medicine and neuroscience. APA assisted with analysis and preparation results. EA, APA and PMM wrote the first draft of the manuscript, and all authors contributed to the final version.

Funding This work was supported by the National Institute of Nursing Research (grant number NR017435) and the National Heart, Lung, and Blood Institute (grant number HL13556), and the National Center for Advancing Translational Science (NCATS) UCLA CTSI (grant number UL1TR001881).

\section{Competing interests None declared.}

Patient and public involvement Patients and/or the public were not involved in the design, or conduct, or reporting, or dissemination plans of this research.

Patient consent for publication Not required.

Ethics approval The procedures were approved by the University of California Los Angeles (UCLA) Institutional Review Board. Explicit consent and patient and public involvement were not possible given the retrospective nature of the analysis, and because the data were electronic medical records. The ethics approval included a waiver of informed consent, and the UCLA Clinical and Translation Science Institute staff extracted and anonymised the data from the electronic health records which were provided to the research team.

Provenance and peer review Not commissioned; externally peer reviewed. Data availability statement Data are available at the following public, open access repository at Harvard Dataverse: https://doi.org/10.7910/DVN/ZY1LDT.
Open access This is an open access article distributed in accordance with the Creative Commons Attribution Non Commercial (CC BY-NC 4.0) license, which permits others to distribute, remix, adapt, build upon this work non-commercially, and license their derivative works on different terms, provided the original work is properly cited, appropriate credit is given, any changes made indicated, and the use is non-commercial. See: http://creativecommons.org/licenses/by-nc/4.0/.

ORCID iD

Paul M Macey http://orcid.org/0000-0003-4093-7458

\section{REFERENCES}

1 Peppard PE, Young T, Barnet JH, et al. Increased prevalence of sleep-disordered breathing in adults. Am J Epidemiol 2013;177:1006-14.

2 Gonzaga C, Bertolami A, Bertolami M, et al. Obstructive sleep apnea, hypertension and cardiovascular diseases. $J$ Hum Hypertens 2015;29:705-12.

3 Peppard PE, Young T, Palta M, et al. Prospective study of the association between sleep-disordered breathing and hypertension. $N$ Engl J Med 2000;342:1378-84.

4 Jonas DE, Amick HR, Feltner C, et al. Screening for obstructive sleep apnea in adults: evidence report and systematic review for the US preventive services Task force. JAMA 2017;317:415-33.

5 Mokhlesi B, Finn LA, Hagen EW, et al. Obstructive sleep apnea during REM sleep and hypertension. Results of the Wisconsin sleep cohort. Am J Respir Crit Care Med 2014;190:1158-67.

6 Senaratna CV, Perret JL, Lodge CJ, et al. Prevalence of obstructive sleep apnea in the general population: a systematic review. Sleep Med Rev 2017;34:70-81.

7 Fryar CD, Ostchega Y, Hales CM, et al. Hypertension prevalence and control among adults: United States, 2015-2016. NCHS Data Brief 2017;289:1-8.

8 Sleep-Related breathing disorders in adults: recommendations for syndrome definition and measurement techniques in clinical research. The report of an American Academy of sleep medicine Task force. Sleep 1999;22:667-89.

9 Berry RB, Budhiraja R, Gottlieb DJ, et al. Rules for scoring respiratory events in sleep: update of the 2007 AASM manual for the scoring of sleep and associated events. deliberations of the sleep apnea definitions Task force of the American Academy of sleep medicine. $J$ Clin Sleep Med 2012;8:597-619.

10 Macey PM. Days between diagnosis of obstructive sleep apnea and hypertension, 2019.

11 Bosc LVG, Resta T, Walker B, et al. Mechanisms of intermittent hypoxia induced hypertension. J Cell Mol Med 2010;14:3-17.

12 Franklin KA, Lindberg E. Obstructive sleep apnea is a common disorder in the population-a review on the epidemiology of sleep apnea. J Thorac Dis 2015;7:1311-22.

13 Suratt PM, Wilhoit SC, Hsiao HS, et al. Compliance of chest wall in obese subjects. J Appl Physiol Respir Environ Exerc Physiol 1984;57:403-7.

14 Ostchega Y, Hughes JP, Terry A, et al. Abdominal obesity, body mass index, and hypertension in US adults: NHANES 2007-2010. Am J Hypertens 2012;25:1271-8.

15 Guerre-Millo M. Adipose tissue and adipokines: for better or worse. Diabetes Metab 2004;30:13-19.

16 Eliasson AH, Kashani MD, Howard RS, et al. Fatigued on venus, sleepy on Mars-gender and racial differences in symptoms of sleep apnea. Sleep Breath 2015;19:99-107.

17 Macey PM, Kumar R, Yan-Go FL, et al. Sex differences in white matter alterations accompanying obstructive sleep apnea. Sleep 2012;35:1603-13.

18 Mirer AG, Young T, Palta M, et al. Sleep-Disordered breathing and the menopausal transition among participants in the sleep in midlife women study. Menopause 2017;24:157-62.

19 Bixler EO, Vgontzas AN, Lin HM, et al. Prevalence of sleepdisordered breathing in women: effects of gender. Am J Respir Crit Care Med 2001;163:608-13.

20 Torres G, Sánchez-de-la-Torre M, Barbé F. Relationship between OSA and hypertension. Chest 2015;148:824-32. 